\title{
New Experimental Investigation of Magnetic and Electric Fields in the Vicinity of High-Voltage Power Lines
}

\author{
Said Ghnimi ${ }^{1 *}$, Adnen Rajhi ${ }^{2}$ and Ali Gharsallah ${ }^{1}$ \\ ${ }^{1}$ Sciences Faculty of Tunis, university Tunis El Manar, 2092, Tunis, Tunisia \\ ${ }^{2}$ Higher School of Technology and Computer Science, University of Carthage, Tunisia
}

(Received 9 November 2015, Received in final form 21 December 2015, Accepted 22 December 2015)

\begin{abstract}
In this paper, the theoretical and experimental characteristics of magnetic and electric fields in the vicinity of high voltage lines are investigated. To realize these measurements and calculations, we have developed some equations for two overhead power line configurations of $150 \mathrm{kV}$ (single circuit, double circuit), based on Biotsavart law, image and Maxwell theories, in order to calculate the magnetic and electric fields. The measurements were done to a maximum distance from the tower of $50 \mathrm{~m}$, at a height of $1 \mathrm{~m}$ from the ground. These experiments take into consideration the real situations of the power lines and associated equipment. The experimental results obtained are near to that of the Biot-Savart theoretical results for a far distance from the tower; and for a distance close to the power line, the results from the image theory are in good agreement with the experimental results.
\end{abstract}

Keywords : magnetic field, electric field, biot-savart law, overhead power lines

\section{Introduction}

For several decades, many researchers have studied the complex electric and magnetic fields within the environment surrounding power lines [1-34]. These lines and their associated equipment, produce the field strength in their proximity, which is likely to have dangerous effects on their environment [1-4].

In the recent years, different methods have been proposed for analyzing the characteristic of these fields for different systems. Many researchers have focused on the theoretical approach of electromagnetic fields to derive a formula for the electric and magnetic fields vector, which is accurate at any distance from the power lines [5-21]; Laforest [8] presented theoretical models based on the charge distribution to calculate the electric field and based on the Biot-Savart law to calculate the magnetic field.

Olsen, in [9], presents the components of magnetic flux density due to an overhead line which carries a phase current using Gauss methods; in [10], the image theories for wires were developed; and [11] gives an approximate answer regarding the characteristics of low frequency

CThe Korean Magnetics Society. All rights reserved.

*Corresponding author: Tel: 0021658828350

Fax: 0021671871666, e-mail: said.ghnimi@gmail.com electric and magnetic fields in the vicinity of power lines.

George Filippopoulos in [12], developed the analytical formula of the magnetic field vector produced by any power line such as a single circuit line (flat, vertical, or delta configurations), as well as for hexagonal lines considered as double circuit lines (six-phase lines).

These analytical methods must be approved by several experimental studies of electromagnetic fields in the proximity of power lines and their associated equipment, such as stations and sub-stations of various voltage levels.

Among the published works [22-36], Misakian [25] gives a survey of the instrumentation measurement methods and standards which can be used to characterize extremely low frequency (ELF) electric and magnetic fields. These methods were employed for measurements of these fields near high voltage lines and their associated equipment. An initial series of measurements were carried out by Farag [26] to determine the levels of EMF in various indoor and outdoor environments (power transmission lines, power cables, substations, domestic electrical equipment) associated with occupational and residential exposure. Feero in [27] presented a measurement of magnetic field profiles around a specific substation, and has verified that adding a substation under an existing transmission line does not increase the magnetic field beyond the substation boundary. In [28], Daily measured and 
calculated the magnetic fields generated by a distribution substation, taking into account currents in the grounding systems, overhead ground wires, and induced currents in equipment structures and ground grid loops.

Safigianni, in [29, 30], examined the $50 \mathrm{~Hz}$ electric and magnetic fields caused by the operation of indoor and outdoor power distribution substations. Jaekel, in [31], makes measurements of the field strength in a combined cycle gas turbine power station for both the steady-state situation and the process of starting the generator. In [32], Kaune analyzed the magnetic field produced by power lines at relatively large distances in comparison to their phase spacing. In [33], researchers conducted the measurement of electric and magnetic fields for conventional three-phase power transmission lines and parallel quadruple high voltage power lines. These values of the extremely low frequency magnetic field are estimated in [34] with a simple model based on the Biot-Savart law, considering the actual shape, the geometry and the conductor's mutual position. This model is verified by measuring these fields on the different overhead power lines.

However, most researchers have not studied the correlation of the characteristics of these fields in different situations, with different equipment, and for several overhead power lines configurations at the same time. To overcome this limitation, we developed this study to determine the level of electric and magnetic fields generated by high voltage overhead lines in our immediate electrical network (STEG), and elaborate these electromagnetic fields, to minimize the degree of disturbance in the vicinity of $150 \mathrm{kV}$ electric power lines.

This paper is organized as follows. In section II, a theoretical approach for electric and magnetic fields in the vicinities of high voltage lines is presented; in section III, we have presented the results and discussion of the experimental investigation of electric and magnetic field characteristics for $150 \mathrm{kV}$ overhead power lines; section IV summarizes all the results of this study by giving some recommendations for further research.

\section{Theoretical Formulation}

A simple method to approximate the magnetic field generated by overhead power lines (Fig. 1) is usually by evaluation using Biot-Savart's law; in phasor notation, the formula for the magnetic field vector, created by a single straight wire conductor $i$ carrying current $\bar{I}_{i}$, in the direction of the y-axis, is given by (1) by supposing that the line is infinite,

$$
\overrightarrow{\bar{B}}_{i}=\mu_{0} \frac{\overrightarrow{\bar{I}}_{i} \times \vec{R}_{i}}{2 \pi R_{i}^{2}}
$$

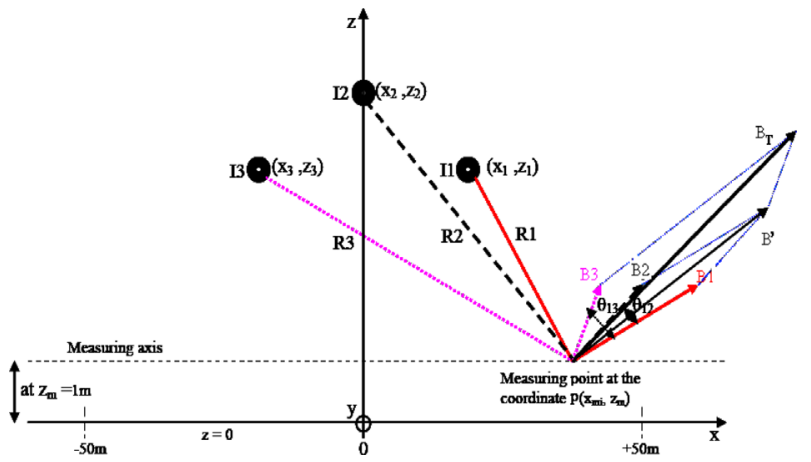

Fig. 1. (Color online) Three-phase configuration (single circuit).

where $\mu_{0}$ represents the permeability of free space; the vector $\overrightarrow{\bar{I}}_{i}=\bar{I}_{i} \vec{e}_{y}$ denotes the current phasor $\overrightarrow{\bar{I}}_{i}$ while $\vec{e}_{y}$ is the unit vector in the direction of the y-axis; $\vec{R}_{i}$ is the vector distance from the conductor to the point of interest $\mathrm{P}$; and the symbol $\times$ denotes the cross product of the vectors $\vec{e}_{y}$ with $\vec{R}_{i}$.

Figure 1 presents the experimental three phase power line configuration with the different real dimensions, and shows the different orientations of the generated magnetic fields from each phase line by taking into account the measurements of angles $\theta_{12}$ and $\theta_{13}$; and in order to measure the total magnetic field generated by the three phase power lines, we considered a vector superposition of the fields created from the different single straight currents. This procedure is rather simple, but the related calculations are rather complex, involving a composition of both vector and phasor quantities.

Therefore, the considered overhead power line with $\mathrm{N}$ conductors produces a magnetic field $\mathrm{B}$ at the measuring point $\mathrm{P}$, and it is considered as the sum of the fields produced by each conductor separately, given by the expression (2), which is the arithmetic calculation of the magnetic field vector.

$$
\overrightarrow{\vec{B}}=\frac{\mu_{0}}{2 \pi} \sum_{i=1}^{N} \frac{\overrightarrow{\bar{I}}_{i} \times \vec{R}_{i}}{R_{i}^{2}}
$$

We consider the orthogonal basis $\left(\vec{e}_{x}, \vec{e}_{y}, \vec{e}_{z}\right)$ and the amplitude of the magnetic field $B_{i}$, for an infinite conductor carrying the current with Ii as the root mean square value, which is given by

$$
\left|B_{i}\right|=\mu_{0} \frac{I_{i}}{2 \pi R_{i}}
$$

With

$$
R_{i}=\sqrt{\left(x-x_{i}\right)^{2}+\left(y-y_{i}\right)^{2}+\left(z-z_{i}\right)^{2}}
$$

In the classical space vector representation, the magnetic 
field vector is expressed by (5);

$$
\overrightarrow{\bar{B}}_{i}=\bar{B}_{i x} \vec{e}_{x}+\bar{B}_{i y} \vec{e}_{y}+\bar{B}_{i z} \vec{e}_{z}
$$

Figure 1 shows the magnetic field $\overrightarrow{\bar{B}}_{i}$ at the field point "P belonging in the x-z plane $\left(\bar{B}_{i,}=0\right)$. The components $\bar{B}_{i x}$ and $\bar{B}_{i z}$ of a single wire conductor $\mathrm{i}$ at the field point $\mathrm{P}$ are given by the following expressions (6) and (7),

$$
\begin{aligned}
& \bar{B}_{i x}=B_{i x}^{r e}+j B_{i x}^{i m} \\
& \bar{B}_{i z}=B_{i z}^{r e}+j B_{i z}^{i m}
\end{aligned}
$$

$B_{i}^{r e}$ and $B_{i}^{i m}$ represent respectively the real and imaginary parts of a complex vector $\overrightarrow{\bar{B}}_{i}$ corresponding respectively to the real and imaginary $I_{i}^{r e}$ and $I_{i}^{i m}$ of the current $\bar{I}_{i}$; however, $\overrightarrow{\bar{B}}_{i}$ is expressed by;

$$
\overrightarrow{\bar{B}}_{i}=\left(B_{i x}^{r e} \vec{e}_{x}+B_{i z}^{r e} \vec{e}_{z}\right)+j\left(B_{i x}^{i m} \vec{e}_{x}+B_{i z}^{i m} \vec{e}_{z}\right)
$$

The components of the total magnetic field $B$ at the point $P\left(x_{m i}, z_{m}\right)$ are given by (9) and (10),

$$
\begin{aligned}
& \bar{B}_{x}=\sum_{i=1}^{N}\left(B_{i x}^{r e}+j B_{i x}^{i m}\right) \\
& \bar{B}_{z}=\sum_{i=1}^{N}\left(B_{i z}^{r e}+j B_{i z}^{i m}\right)
\end{aligned}
$$

And the resultant RMS value of the total magnetic field is given by expressions (11) and (12),

$$
\begin{aligned}
& B=\sqrt{B_{x}^{2}+B_{z}^{2}} \\
& B=\sqrt{\left(B^{r e}\right)^{2}+\left(B^{i m}\right)^{2}}
\end{aligned}
$$

For a three-phase system with straight parallel conductors of infinite length (Fig. 1),

$$
B=\sqrt{\left(\sum_{i=1}^{3} B_{i x}^{r e}\right)^{2}+\left(\sum_{i=1}^{3} B_{i z}^{r e}\right)^{2}+\left(\sum_{i=1}^{3} B_{i x}^{i m}\right)^{2}+\left(\sum_{i=1}^{3} B_{i z}^{i m}\right)^{2}}
$$

Table 1 summarizes the variation of Ri, $\theta_{12}$ and $\theta_{13}$ with the measuring $x_{m}$ for $200 \mathrm{~A}$ as the line intensity current.

We note that the angles $\theta_{12}$ and $\theta_{13}$ become important when we are near the line, therefore some attention should be paid to the orientation of the apparatus in order to measure the experimental electric and magnetic fields with accuracy.

Concerning the theoretical approach of the produced electric field, there are two main methods according to the literature; one is based on the charge distribution and the use of the image concept, and the other is based on the coupled relation of the electric and magnetic field, which requires a solution of the Maxwell equation.

For the first method, the expression of the electric field is given by Olsen [10] and Malik [35],

$$
[P] \cdot[Q]=[V]
$$

With $[P],[Q]$ and $[V]$ present, the $(\mathrm{N} \times \mathrm{N})$ matrix of the potential coefficients, and the complex vectors of charge and potential of the dimension $\mathrm{N}$ for a system with $\mathrm{N}$ conductors placed above the ground.

The electric field is calculated according to the following equation,

$$
\vec{E}=-\operatorname{grad} V
$$

For the second method, the well-known coupled electric and magnetic field relation is given by the MaxwellFaraday equation (16),

$$
\vec{\nabla} \times \vec{E}=-\frac{\partial \vec{B}}{\partial t}=\left(\frac{\partial E_{y}}{\partial z} \vec{e}_{x}-\frac{\partial E_{y}}{\partial x} \vec{e}_{z}\right)
$$

where $\vec{e}_{x}$ and $\vec{e}_{z}$ are the unit vectors along the $x$ and $z$ axes, perpendicular to axis $y$.

$$
\left(\frac{\partial E_{y}}{\partial z} \vec{e}_{x}-\frac{\partial E_{y}}{\partial x} \vec{e}_{z}\right)=-j \omega \vec{B}
$$

With

$$
\left(\frac{\partial E_{y}}{\partial z} \vec{e}_{x}-\frac{\partial E_{y}}{\partial x} \vec{e}_{z}\right)=-j \omega\left(B_{x} \vec{e}_{x}+B_{z} \vec{e}_{z}\right)
$$

The total electric field at the observation point $P$, is approximated by integrating (18) and the resultant electric field $\bar{E}$, and is given by the following equation:

Table 1. Magnetic field dependency with the distance $\mathrm{x}$.

\begin{tabular}{ccccccccc}
\hline \hline $\mathrm{x}(\mathrm{m})$ & -90 & -50 & -30 & -10 & 10 & 30 & 50 & 90 \\
\hline $\mathrm{R} 1(\mathrm{~m})$ & 97.0 & 58.9 & 41.2 & 27.0 & 23.7 & 34.5 & 51.2 & 88.7 \\
$\mathrm{R} 2(\mathrm{~m})$ & 93.3 & 55.7 & 38.7 & 26.5 & 26.5 & 38.7 & 55.7 & 93.3 \\
$\mathrm{R} 3(\mathrm{~m})$ & 88.7 & 51.2 & 34.5 & 23.7 & 27.0 & 41.2 & 58.9 & 97.0 \\
$\theta_{12}(\mathrm{deg})$ & 1.57 & 3.22 & 5.46 & 9.65 & 8.08 & 2.41 & 0.49 & -0.27 \\
$\theta_{13}(\mathrm{deg})$ & 1.30 & 3.71 & 7.88 & 17.7 & 17.7 & 7.88 & 3.71 & 1.30 \\
\hline
\end{tabular}




$$
\bar{E}=j \omega \sqrt{\left(\sum_{i=1}^{3} \int_{C_{i}} \overrightarrow{B_{i x}} \cdot \overrightarrow{d l}\right)^{2}+\left(\sum_{i=1}^{3} \int_{C_{i}} \overrightarrow{B_{i z}} \cdot \overrightarrow{d l}\right)^{2}}
$$

The theoretical simulation process of the electric field near the high voltage power lines can be calculated more accurately with the first method, because the electrostatic concept is more important; but when we consider the measuring points far away from the line, the concept of coupled electric and magnetic fields can be used.

\section{Results and Discussion}

The different methods presented in the literature often require an accurate measurement model of the amplitude of electric and magnetic field intensities.

To detect the values of these fields for two considered geometrical configurations (single circuit, double circuit), measurements were made at various hours with current intensity varying between 178A and 196A, as shown in Fig. 2.

The measurements of electric and magnetic fields were performed in three different situations (M1, M2, and $\mathrm{M}_{\mathrm{N}-1, \mathrm{~N}}$ ); and we took measurements along the direction ( $\left.x^{\prime} \mathrm{Ox}\right)$, as shown in Fig. 3.

The results of the electric and magnetic fields according to distance were obtained by the measuring apparatus $<<$ HI-3604 ELF Field Strength Measurement System $>>$ (Fig. 4), and had the following characteristics:

i) Frequencies: The evaluation of electric and magnetic fields associated with $50 / 60 \mathrm{~Hz}$.

ii) Sensitivity:

- Electric fields $1 \mathrm{~V} / \mathrm{m}-199 \mathrm{KV} / \mathrm{m}$.

- Magnetic fields 0, $01 \mathrm{mT}-2000 \mathrm{mT}$.

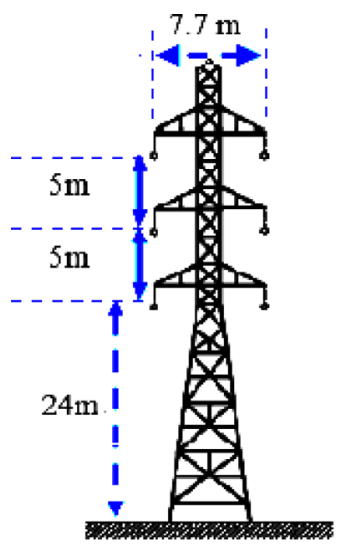

(a)

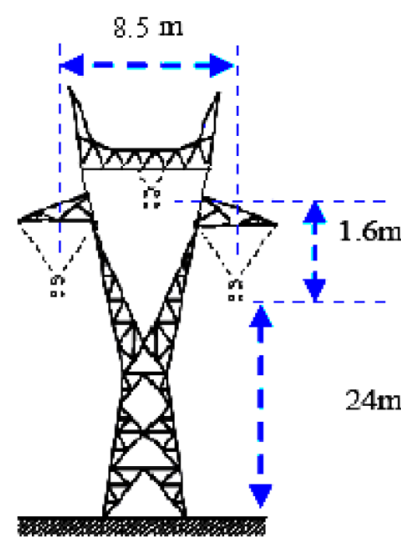

(b)
Fig. 2. (Color online) Cross section of the power lines: (a) double circuit, (b) single-circuit.

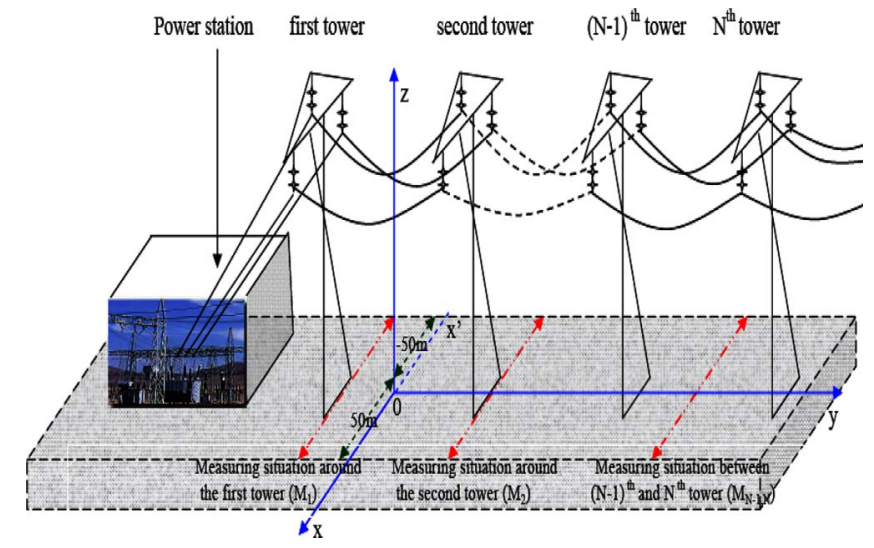

Fig. 3. (Color online) The measuring configuration of the electric and magnetic fields of the $150 \mathrm{kV}$ power.

iii) Sensors: There are two switch-selectable sensors for measuring both electric and magnetic fields.

iiii) Polarization Reponses: displacement sensors and magnetic field sensors are designed for response to one field polarization component at a time.

The effects of the angles $\theta_{12}$ and $\theta_{13}$, described in section 2 , are taken into consideration due to the orientation of the apparatus relative to the ground.

The objective of this experimental investigation is to prove the validity of the above model and its parameters in order to calculate the amplitude level of the magnetic and electric fields.

The theoretical results of the magnetic and electric fields were produced using the Matlab environment and the Biot-Savart law (eq. 13) to determine the magnetic field and the calculation of the electric field using two concepts:

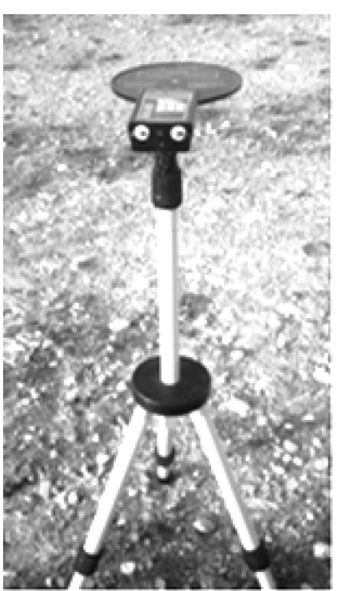

(a)

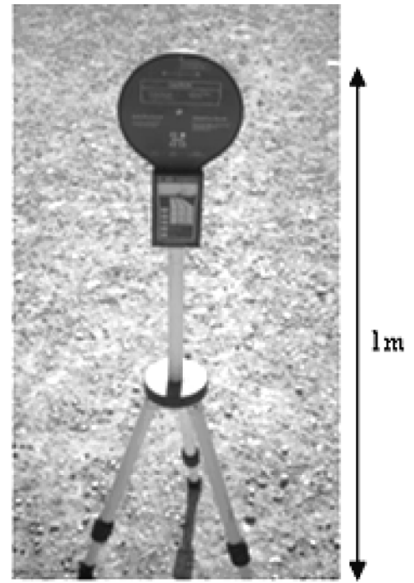

(b)
Fig. 4. The measuring setup of the 3604 ELF system: (a) for E-field; and (a) for B-field. 

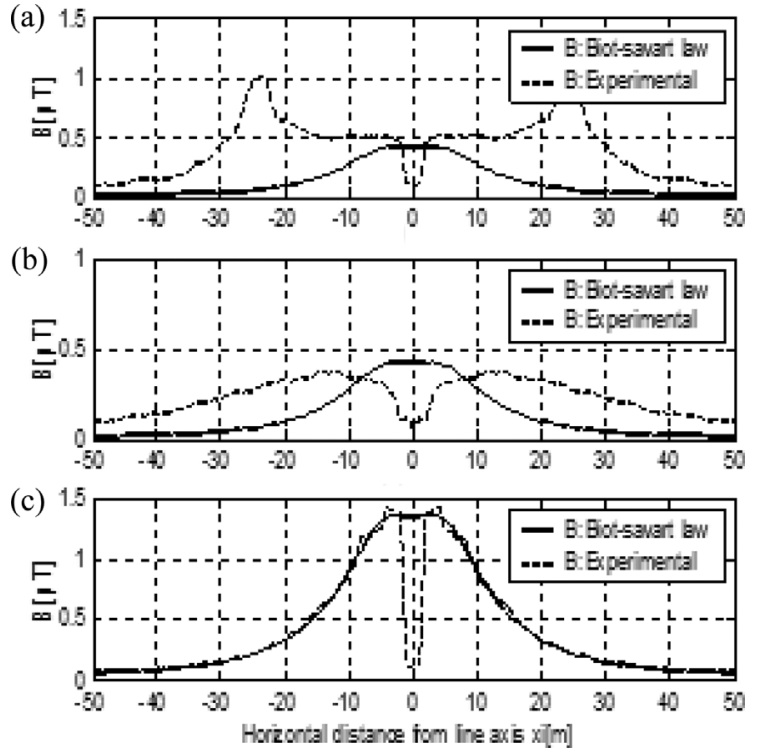

Fig. 5. Theoretical and experimental results of the magnetic field for the single circuit configuration; (a) for measuring situation M1, (b) for measuring situation M2, (c) for measuring situation $\mathrm{M}_{\mathrm{N}-1, \mathrm{~N}}$.

- The concept of the uncoupled electric and magnetic fields (image theory) (eq. 15).

- The concept of the coupled electric and magnetic fields (Maxwell theory) (eq. 19).

In order to measure experimentally the magnetic and electric fields, we used the experimental setup of Fig. 3 and Fig. 4 for the single and double circuit configuration of $150 \mathrm{KV}$ power lines. For each configuration, the measuring apparatus were placed along the x'ox axis for three different measuring situations (M1, M2, $\mathrm{M}_{\mathrm{N}-1, \mathrm{~N}}$ ).

In the theoretical calculations shown in Fig. 5 and Fig. 6 , for the measuring situations M1 and M2, the considered height of the tower is $24 \mathrm{~m}$, but for the $\mathrm{M}_{\mathrm{N}-1, \mathrm{~N}}$ measuring situation, we take into consideration that the power lines are not parallel to the ground, since they have a parabolic wire configuration; therefore, the considered height for this measuring situation is $16 \mathrm{~m}$.

The dependency of the experimental magnetic and electric fields on the distance from the power line is shown in Fig. 5 to Fig. 8 for single circuit and double circuit power lines. We note that there is good agreement between the experimental and theoretical results for both configurations when the measuring point is far from the lines; but at the proximity of the lines there are appreciable errors from the theory model, which can be explained by the presence of the field created from the power station.

In Fig. 5a the magnetic field variation in the intervals $[-30,-20]$ and $[20,30]$ increase to the maximal value in
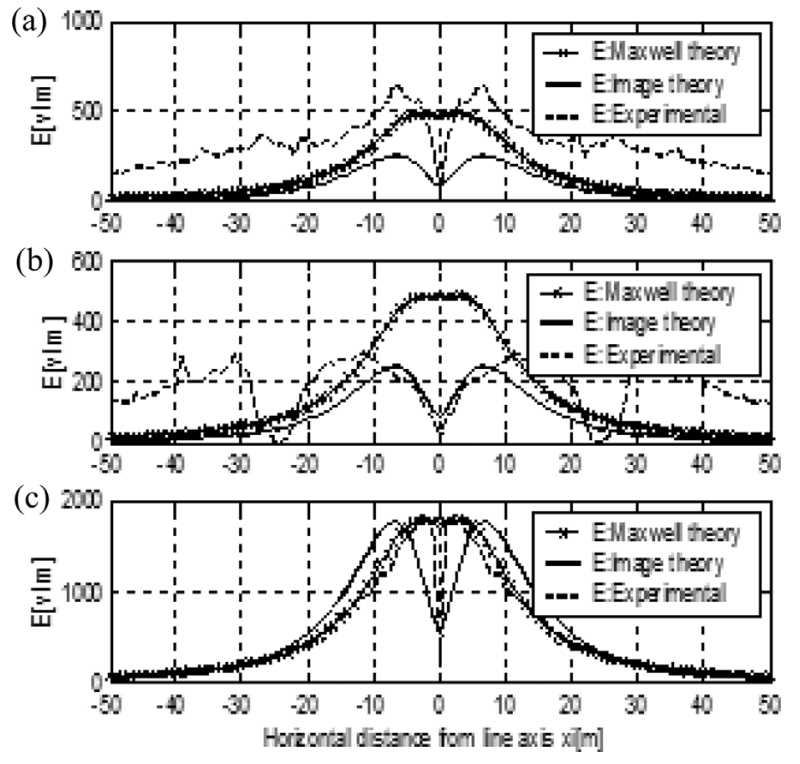

Fig. 6. Theoretical and experimental results of the electric field for the single circuit configuration; (a) for measuring situation M1, (b) for measuring situation M2 (c) for measuring situation $\mathrm{M}_{\mathrm{N}-1, \mathrm{~N}}$.

the measured point M1, then the electric field at M2 in Fig. $6 \mathrm{~b}$ decreases for the minimum value in the same interval. Indeed, in the literature the power lines are always considered as infinitely long lines. However, in reality there are physical ends between the power station and the M1 measuring situations. These physical ends
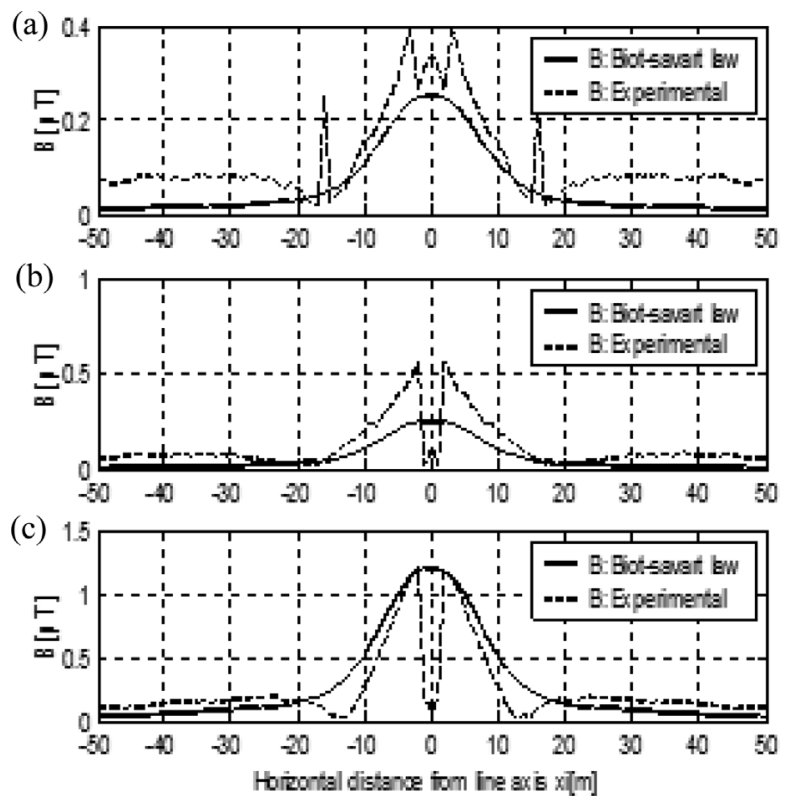

Fig. 7. Theoretical and experimental results of the magnetic field for the double circuit configuration; (a) for measuring situation M1, (b) for measuring situation M2, (c) for measuring situation $\mathrm{M}_{\mathrm{N}-1, \mathrm{~N}}$. 

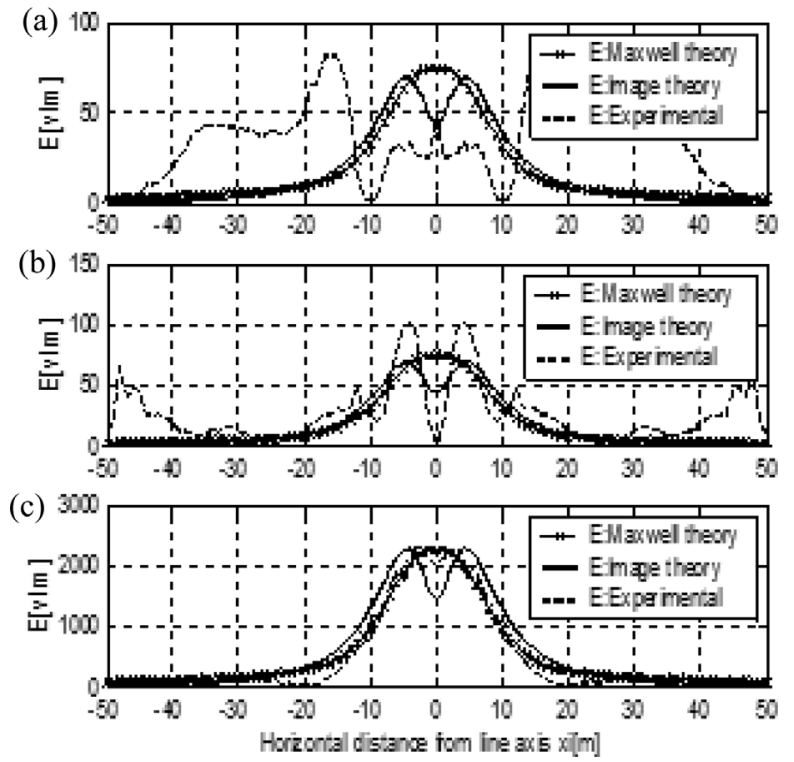

Fig. 8. Theoretical and experimental results of the electric field for the double circuit configuration; (a) for measuring situation M1, (b) for measuring situation M2, (c) for measuring situation $\mathrm{M}_{\mathrm{N}-1, \mathrm{~N}}$.

may influence the calculation of electric and magnetic fields.

The results shown in the previous figures allowed us to confirm the validity of the developed model, and also confirm the greater errors between the theoretical and experimental results produced in the measuring situation $M_{1}$ (Fig. 7a and Fig. 8a), which are due to the existence of the power station.

These errors are attributed not only to the power line configuration, but are also related to the measuring process itself, since the current intensities are changing during the measurements.

On the other hand, we note that the variation of the magnetic and electric fields along the line is very significant in the measuring situation $\mathrm{M}_{\mathrm{N}-1, \mathrm{~N}}$. The position of power line equipment presents a complexity in the magnetic and electric fields for different situations. The field characteristics in these environments, which are far from power lines, can have significant differences when the measuring point is near to ground level. The variation of these fields is due to the location and nature of the line, creating a non-uniform distribution of the harmonics; this affects the measuring values of the instruments (calibration procedures and measurement techniques). The more complex nature of the fields away from power lines also limits the usefulness of the proposed theories in the literature.

These theories indicate that these fields decrease as soon as we are far from the line. However, there are special regions where these fields are more significant for a distance $x>0$.

Indeed, experimental analysis reveals more complicated variations of this field with the distance $x$ in Fig. 5 and Fig. 7. This may be a sign of the limitation of the theories exposed above.

The magnetic field peak for the intervals $[-20-10]$ and [10 20] at the measuring point $M_{1}$ explain the delay of the single circuit as compared with the double circuit (Fig. 5a and Fig. 7a); these results prove that the field's variation is slow in the first case when compared with the second case, and is explained by the difference in the conductor numbers.

Olsen [1] presented the electric and magnetic fields as coupled, and has also shown that there are some circumstances under which they are uncoupled; this is often the case for fields created by $50 / 60 \mathrm{~Hz}$ power lines because they vary so slowly in time that Maxwell's equations almost become decoupled into electrostatic and magnetostatic fields.

The results shown in Fig. 9 and Fig. 10 present the magnetic and electric field variations according to the distance $x$, from which we can conclude that if we have an increase in magnetic field amplitude, we do not automatically have the increase in electric field; this can be explained by the uncoupled concept of the electric and magnetic fields.

Since we are this study is focused on the effect of power lines on equipment located in the vicinity of these lines, Fig. 11 and Fig. 12 present the amplitude of the magnetic and electric fields for the different measuring situations, and for both configurations at distance $x$. The
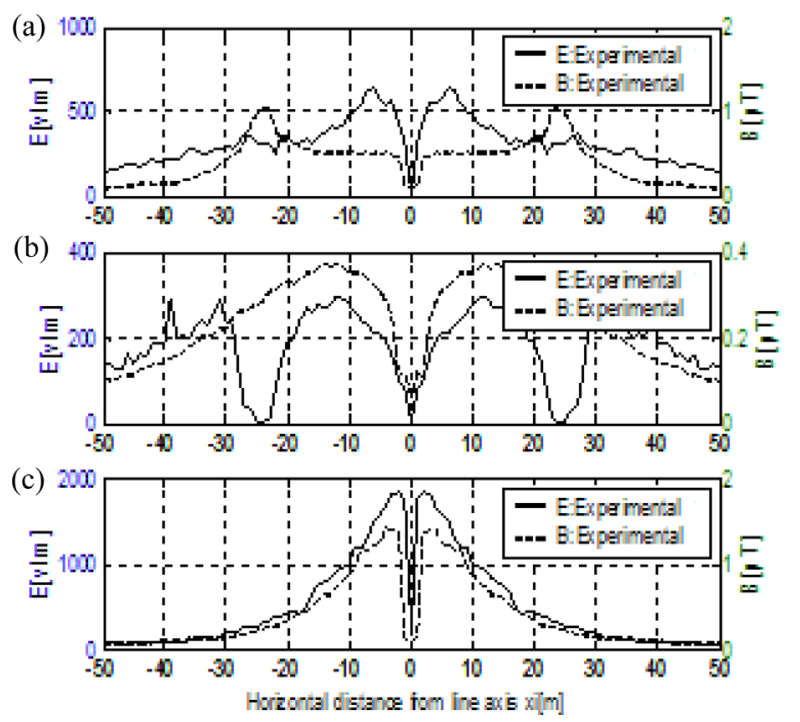

Fig. 9. (Color online) Magnetic and Electric field intensities for the single circuit configuration in the different measuring situations: (a) M1, (b) M2, (c) $\mathrm{M}_{\mathrm{N}-1, \mathrm{~N}}$. 

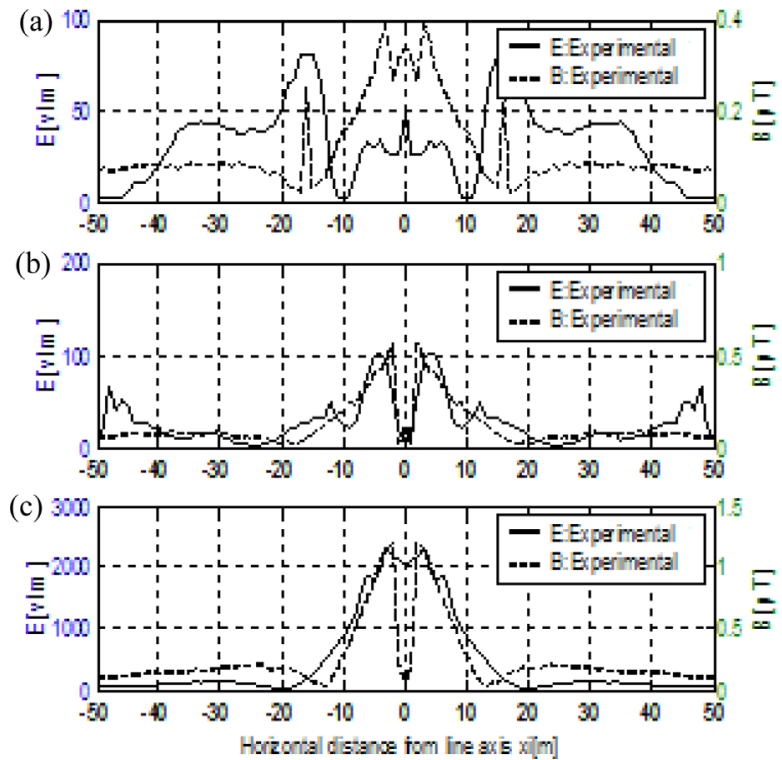

Fig. 10. (Color online) Magnetic and electric fields intensities for the double circuit configuration in the different measuring situations: (a) M1, (b) M2, (c) $\mathrm{M}_{\mathrm{N}-1, \mathrm{~N}}$.
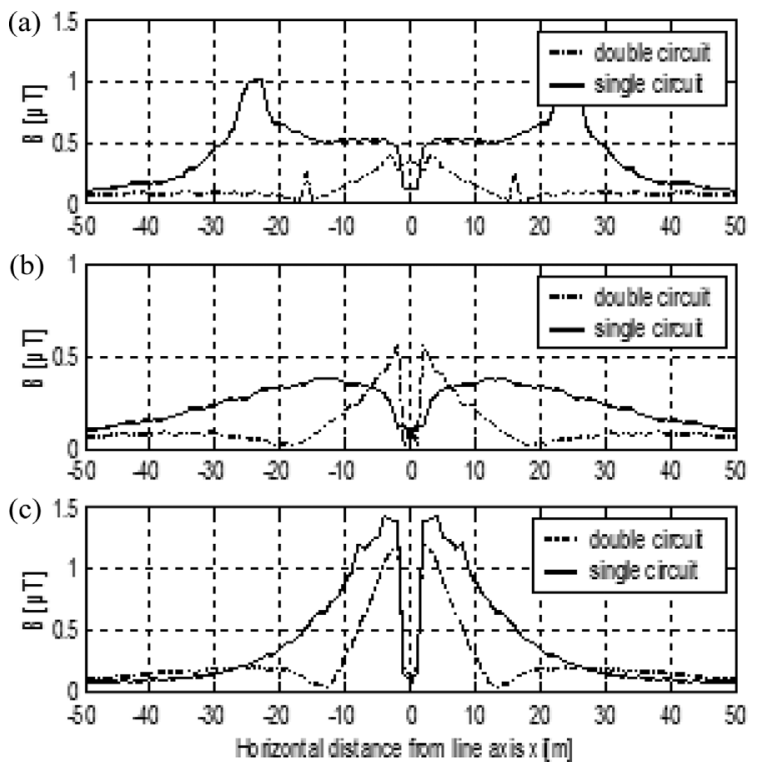

Fig. 11. Distribution of the magnetic field intensity generated by single and double circuit power lines.

electric and magnetic fields do not have the same behavior, which explains why they depend on the voltage and the current sources.

The dependency of the magnetic and electric field intensities with the phase number of the power line is given in Fig. 11 and Fig. 12; these results show a proportional slight of these fields with the phase number.

We can distinguish from the plots shown in Fig. 11 that the magnetic field associated with the single circuit in the
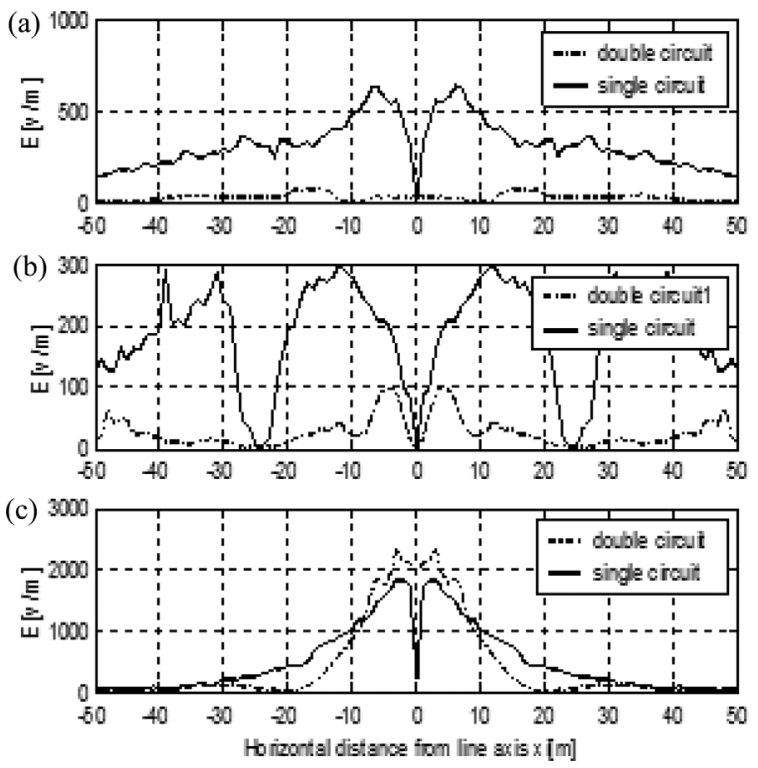

Fig. 12. Distribution of the electric field intensity generated by single and double circuit power lines.

proximity of the power station and nearby the power lines (i.e., Measurement points M1 and M2) is lower than that of the double circuit. However, if we are far away from the power line and the power station, B becomes more important as compared with the magnetic field associated with the double circuit.

Figure 12 shows that at the distance far from the power station and near to the power lines $\left(\mathrm{M}_{\mathrm{N}-1, \mathrm{~N}}\right)$, the electric field of the double circuit becomes intensifies as compared to that of the single circuit configuration, which explains why the electric field depends on the number of conductor wires only in the far fields corresponding to the measurement points $\mathrm{M}_{\mathrm{N}-1, \mathrm{~N}}$.

In spite of the electric field, the magnetic field is dependent on the number of conductor wires only in the vicinity of the power station.

\section{Conclusions}

The main aim of this paper was to present the measurements of magnetic and electric fields produced by $150 \mathrm{kV}$ overhead power lines for two configurations integrated in the network of the Tunisian Company of Electricity and Gas; and the experiments carried out for each measuring situation along the line can be employed in the support of the planning of the electrical systems within Tunisia.

The experimental results showed the characteristics of these fields in different positions of the power lines, and elaborate the electromagnetic concept for their environment. This study has two significant goals which were 
met: (i) verification of the validity of the results obtained with the Biot-Savart law, image and Maxwell theories; and (ii) checking of the disturbance degree and limitation of the electric and magnetic fields in the indicated environment.

The theoretical and experimental results are not particularly similar in the measuring situation $\mathrm{M} 1$, and this limitation is due to the fact that we did not introduce the effect of the associated equipment. Therefore, to improve this theoretical approach we have to take into consideration the image theory near the power line and the BiotSavart law for situations far from the line. The limit does not exceed the degree of compliance with international regulations $(5 \mathrm{KV} / \mathrm{m}$ for the electric field and $100 \mu \mathrm{T}$ for the magnetic field) in the region of north Tunisia.

Future projects could revise the theoretical formulation of these fields and include the effect of the overhead power line configuration and its associated equipment on the environment.

\section{Acknowledgements}

The authors wish to acknowledge that this work has been carried out with the help of the Tunisian Company of Electricity and Gas of Tunis, and we make a point of thanking Mr. Chaari Mohamed, Athimni Ahmed, Zommiti Mohamed, Abadlia Abderrazek, Ben Jemma Habib and Bel Hadj Sghair Ali, for their moral assistance and their encouragement.

\section{References}

[1] G. Kulkarni and W. Z. Gandhare, ACEEE Int. J. on Electrical and Power Engineering 3, 28 (2012).

[2] D. Djalel and M. Mourad, Journal of Electrical and Electronic Engineering 2, 1 (2014).

[3] B. A. Rachedi, A. Babouri, A. Lemzadmi, M. Nemamcha, and F. Berrouk, IEEE International Conference on Intelligent Energy and Power Systems 36 (2014).

[4] S. Ghnimi, A. Rajhi, A. Gharsallah, and F. Khlifa, Am. J. Appl. Sci. 8, 499 (2011).

[5] G. Duyan, X. Guizhi, Y. Hongli, Y. Shuo, Y. Qingxin, and Y. Weili, $30^{\text {th }}$ Annual International Conference of the IEEE Engineering in Medicine and Biology Society, Canada. 1331 (2008).

[6] L. Xiao and K. E. Holbert, IEEE North American Power Symposium (NAPS). 1 (2014).

[7] M. Milutinov, A. Juhas, and M. Prša, $2^{\text {nd }}$ International Conference on Moderen Power Systems MPS, Romania. 313 (2008).

[8] J. J. Laforest, Transmission line reference Book $345 \mathrm{kV}$ and above, Electric Power Research Institute (1982) pp. $329-417$.
[9] R. G. Olsen, D. Deno, and R. S. Baishiki, IEEE Trans. Power Del. 3, 2127 (1988).

[10] R. G. Olsen and T. A. Pankaskie, IEEE Trans. Power App. Syst. 102, 769 (1983).

[11] R. G. Olsen and P. S. Wong, IEEE Trans. Power Del. 7, 2046 (1992).

[12] G. Filippopoulos and D. Tsanakas, IEEE Trans. Power Del. 20, 1474 (2005).

[13] E. Salinas, Proc. $5^{\text {th }}$ Int. Power Engineering Conf., Singapore. 325 (2001).

[14] D. Tsanakas, E. Mimos, and A. Tzinevrakis, International Conference on Energy \& Environmental Systems, Greece 237 (2006).

[15] P. Pettersson, IEEE Trans. Power Del. 11, 1587 (1996).

[16] C. Garrido, A. F. Otero, and J. Cidrás, IEEE Trans. Power Del. 18, 1310 (2003).

[17] R. G. Olsen, D. Deno, and R. S. Baishiki, IEEE Trans. Power Del. 3, 2127 (1988).

[18] R. G. Olsen and T. A. Pankaskie, IEEE Trans. Power App. Syst. 102, 769 (1983).

[19] A. A. Dahab, F. K. Amoura, and W. S. Abu-Elhaiga, IEEE Trans. Power Del. 20, 2114 (2005).

[20] F. P. Dawalibi, IEEE Trans. Power Delivery 8, 1285 (1993).

[21] P. S. Maruvada, A. Turgeon, D. L. Guolet, and C. U. Cardinal, IEEE Trans. Power Delivery 13, 1322 (1998).

[22] A. O. Sougui and M. Z. Jenu, IEEE Asia-Pacific Conference on Applied Electromagnetics, Malaysia 207 (2014).

[23] T. Lisewski and J. Luszcz, International Symposium on Electromagnetic Compatibility, Sweden. 360 (2014).

[24] P. S. Maruvada, A. Turgeon, D. L. Guolet, and C. U. Cardinal, IEEE Trans. Power Delivery. 13, 1328 (1998).

[25] M. Misakian, IEEE International Symposium on Electromagnetic Compatibility. 150 (1993).

[26] A. S. Farag, M. M. Dawoud, T. C. Cheng, and J. S. Cheng, Elect. Power Syst. Res. 48, 151 (1999).

[27] W. E. Feero, J. Yontz, and J. H. Dunlap, IEEE Trans. Power Del. 4, 1862 (1989).

[28] W. K. Daily and F. Dawalibi, IEEE Trans. Power Del. 9, 324 (1994).

[29] A. Safigianni and C. G. Tsompanidou, IEEE Trans. Power Del. 20, 1800 (2005).

[30] A. Safigianni and C. G. Tsompanidou, IEEE Trans. Power Del. 24, 38 (2009).

[31] B. Jaekel, Proc. Int. Wroclaw Symp. Electromagnetic Compatibility, Poland 133 (1998).

[32] W. T. Kaune and L. E. Zaffanella, IEEE Trans. Power Del. 7, 2082 (1992).

[33] H. Ahmad, N. A. Ahmad, and M. I. Jambak, Proceedings of the $\mathrm{XIV}^{\text {th }}$ International Symposium on High Voltage Engineering, China. 1 (2005).

[34] G. Petrović, T. Kilić, and T. Garma, Elektronika ir Elektrotechnika 19, 33 (2013).

[35] N. H. Malik, IEEE Trans. Electrical Insulation 24, 3 (1989). 